\title{
The effect of a visual indicator on rate of visual search: Evidence for processing control*
}

\author{
JOHN E. HOLMGREN \\ University of Maryland, College Park, Maryland 20742
}

\begin{abstract}
Search rates were estimated from response latencies in a visual search task of the type used by Atkinson, Holmgren, and Juola (1969), in which a S searches a small set of letters to determine the presence or absence of a predesignated target. Half of the visual displays contained a marker above one of the letters. The marked letter was the only one that had to be checked to determine whether or not the display contained the target. The presence of a marker in a display significantly increased the estimated rate of search, but the data clearly indicated that Ss did not restrict processing to the marked item. Letters in the vicinity of the marker were also processed. These results were interpreted as showing that Ss are able to exercise some degree of control over the search process in this type of task.
\end{abstract}

When a $S$ must search through a small set of visually presented letters (five or fewer) to determine the presence or absence of a particular predesignated target letter, response latency increases roughly linearly with the number of letters displayed. Moreover, the slope of the linear function is about the same regardless of whether or not the target item is in the display. This result is most clearly demonstrated by Atkinson, Holmgren, and Juola (1969). The simplest (although not the only) interpretation of this finding is that Ss carry out an exhaustive serial search through the visual display; that is, the display items are compared one at a time with some internal representation of the target item, the comparison process terminating only after all display items have been compared with the target, regardless of whether or not the target appears in the display. Under this interpretation of the data, the slope of the linear latency function is an estimate of the average time necessary to carry out one of these comparisons.

An important question regarding this type of visual information processing is the extent to which the search processes involved are under cognitive control. In particular, can the amount of processing carried out by Ss be reduced under certain circumstances, or in tasks of this type do Ss "automatically" process all items in the visual display? At present, no clear answer to this question exists for "yes-no" visual search tasks of this type (i.e., two-response tasks in which the S's response indicates "yes," the target item was in the display, or "no," it was not).

Most of the evidence pertaining to the question of control in the processing of visual displays comes from experiments in which some variant of the report procedure was used. In these studies, instead of searching a display for a particular target, Ss attempt to

*'This work is based upon a portion of a doctoral dissertation submitted to Stanford University. The research was carried out under a NIMH Terminal Graduate Fellowsh ip and was supported in part by NASA Grant NGR-05-020-244. report the identity of one or more of the items displayed. Studies by Sperling (1960) and Averbach and Coriell (1961) have shown that auditory and visual cues presented during or shortly after the exposure of a display can increase the probability of correctly reporting certain subsets of items in the display. In the Sperling task, the pitch of a tone told the $S$ which row of letters in the display was to be reported. The Averbach and Coriell procedure employed a visual marker above one of the letters in a linear array. The S was required to report only the marked letter. These studies have been interpreted as indicating that Ss are able to use auditory and visual cues to restrict, in some sense, processing of the display to the cued items (e.g., Rumelhart, 1970).

More recently, Eriksen and Hoffman (1972a, b, 1973) have attempted to provide more direct evidence as to the effects of visual cues on processing time by looking at report latencies in a task much like that used by Averbach and Coriell (1961). Among other findings, they showed that a bar marking the letter to be reported resulted in shorter response latencies if it preceded the display by $150 \mathrm{msec}$ than if it appeared simultaneously with the display. They also found that report latency increased with the number of letters in the display, the increase being greater with a simultaneous marker. These results were interpreted as showing that it takes $100 \mathrm{msec}$ or more to attend selectively to the area containing the marked item, with everything within about a 1-deg area centered on the marked item being processed in detail; items outside the 1-deg area were thought to be processed at a more gross level.

With regard to drawing inferences about control of processing in yes-no tasks from these results, there are two problems. First, there are distinct differences between tasks using report and those using yes-no responses. In the various report procedures, Ss must attempt to identify one or more of the items in the display, whereas in yes-no tasks, items are simply classified as matching or not matching the target item. It 
is therefore likely that the processes involved in the two types of tasks are not the same. Second, even if one were to assume that the processes are the same in the two cases, it is difficult, using report paradigms, to determine the extent to which processing is affected by the use of various types of cues. This is due to the fact that in most tasks involving cued report, there is no natural control condition (one involving processing without the cue) against which to compare cued processing.

The purpose of the studies reported below is to investigate the question of control of visual processing by looking at the effects of a marker in a visual search task similar to that used by Atkinson et al (1969). Half of the displays used for the task contain a marker above one of the items; for these displays, Ss need only determine whether or not the marked item matches the target. If Ss are able to use the marker to reduce the number of items searched, then when response latencies are plotted as a function of display size, the slope of the resulting function should be less than if no marker appeared in the display. The extreme result would be for latencies to show no increase at all with display size, indicating that Ss are able to restrict processing to the marked item. If the marker affects the intercept of the latency function but not the slope, this would suggest that Ss do not exercise control over the number of items searched, but rather over some other stage of processing.

\section{EXPERIMENT I}

\section{Method}

Subjects. The Ss for this study were six females obtained through an ad in the campus paper. All had normal vision and were paid $\$ 1.75$ for each session of the experiment.

Apparatus. Visual displays were presented to Ss by means of an Iconix automated two-field tachistoscope. The $S$ was seated at a station consisting of a display panel and a response panel. The display panel, $20 \mathrm{~cm}$ wide and $36 \mathrm{~cm}$ high, was recessed $25 \mathrm{~cm}$ into the box holding the optical equipment. At the bottom of the display panel was mounted an IEE Binaview unit on which could be presented any one of a full set of alphanumeric characters. Above this unit was mounted a ground-glass rear-projection screen. Behind the screen was a black metal plate bearing four small colored lights and a large circular aperture, none of which were visible unless illuminated. The response panel consisted of a board on which were mounted three Johnson code transmitting keys. The centers of the keys fell at 3.8-cm intervals along an arc extending from 290 to 340 deg.

Displays were projected onto the ground-glass screen through the large circular aperture, providing an illuminated area $5.2 \mathrm{~cm}$ in diam. Stimuli were displayed by means of a random-access slide projector modified for use with a special light source (Sylvania glow modulator, No. R1131C). A second projector, optically identical to the first but holding a single slide, served to illum inate the screen between stimulus exposures.

Stimuli. A total of 192 slides were prepared for this study. These were divided into two equal-sized categories referred to as "marker" and "no-marker" slides. The set of 96 no-marker slides consisted of the four display sizes, 2 through 5 . There were 24 slides at each display size. Each of the slides contained a horizontal row of uppercase consonants flanked by dollar signs. The consonants used for each slide were selected randomly with the restriction that, at each display size, each consonant appear at least once in every serial position. The dollar signs served as delimiters to equate for any possible lateral masking effects. A five-letter display subtended a visual angle of about $2 \mathrm{deg}$ at the viewing distance of about $60 \mathrm{~cm}$.

The 96 marker slides were prepared in the same manner as the no-marker slides. However, each slide in this set had a marker above one of the consonants. This marker, when projected, appeared as a black vertical line, $6 \mathrm{~mm}$ long, directly above one of the letters. There was a gap of $3 \mathrm{~mm}$ between the bottom of the line and the top of the letter. With the exception of five-letter displays, all serial positions at each display size were marked equally often. For Display Size 5 , the middle position was marked on four instead of five of the slides.

Procedure. Each trial in this study consisted of the following series of events: (1) Two seconds after the start of the trial, a consonant appeared for $2.5 \mathrm{sec}$ on the Binaview. During this initial 4.5-sec period, an orange light was present directly above the circular aperture if the subsequent display was to contain a marker. (2) Following the offset of the Binaview, and while holding down the center key, the $S$ initiated the display presentation by pressing a button held in her left hand. (3) Exactly $.5 \mathrm{sec}$ af ter pressing the button, the display appeared for $400 \mathrm{msec}$ and two latency counters began counting. (4) Half of the Ss responded by hitting the left response key if the target letter appeared in the display (positive response), and the right key if it did not (negative response). For the other half of the Ss, the response assignments were reversed. Automatically recorded were the S's response, the time from the onset of the display to the release of the center key, and the time from the onset of the display to the hitting of a response key. (5) One of three feedback lights came on for $2 \mathrm{sec}, 1.6 \mathrm{sec}$ after the onset of the display. A green light indicated a correct response, red indicated an error, and white indicated no response within the 1.6-sec response interval.

The projector that illuminated the screen between stimulus exposures contained a slide with four dots arranged in a rectangle. When projected, this rectangle measured $2.2 \times 1.3 \mathrm{~cm}$ and subtended a horizontal visual angle of $2.1 \mathrm{deg}$. The display always appeared centered within this rectangle. Ss were instructed to fixate on the center of the rectangle. The brightness of the pre- and postexposure field was $6.2 \mathrm{~mL}$, as measured by a Macbeth illuminometer; the display brightness was $7.0 \mathrm{~mL}$.

Each of the 10 sessions consisted of eight warm-up trials plus 288 data trials, with a short break half-way through the session. Ss were instructed at the beginning of each session to respond as fast as possible, make as few errors as possible, and to always notice whether or not a display was going to contain a marker.

During the last two sessions for each $S$, the procedure was changed slightly. At the beginning of each marker trial, no cue was given informing the $S$ that the display would contain a marker. This was done in order to determine the extent to which Ss' performance was a function of prior knowledge concerning the type of display to be presented on each trial.

The slide to be shown on each trial was selected randomly, with the restriction that in any one session positive and negative trials occurred equally of ten, as did marker and no-marker trials. Also, for positive no-marker trials at each display size, the target letter appeared equally often in each serial position.

\section{Results}

On each trial, both a "release" and "terminal" latency were recorded. A release latency is the time from the onset of a display to the release of the center key; a terminal latency is the time from display onset to the hitting of a response key. Unless otherwise noted, all results presented below are for terminal latencies.

The data of basic interest in this study are the mean 
latencies of positive and negative correct responses for both marker and no-marker trials as a function of display size. These functions are shown in Fig. 1, averaged across Ss. The data are shown for Sessions 3.8 and separately for Sessions 9 and 10 . Recall that for Sessions 9 and 10 no cue informed Ss as to which trial would contain a marker. There was a relatively substantial drop in mean response latency over the first two sessions, so these were not included in Fig. 1.

Before presenting the results of any statistical analyses, it is worth mentioning some general conclusions based on visual examination of the data for Sessions 3.8 in Fig. 1. It is clear for marker trials that latencies increase roughly linearly with display size and that the rate of increase is less than for no-marker trials; moreover, latencies for positive and negative marker trials increase at about the same rate. For no-marker trials, the negative curve is distinctly nonlinear and shows a greater increase with display size than does the positive curve. In all cases, negative mean latencies are slower than the corresponding positive mean latencies; this is an almost universal result in tasks of this type (Nickerson, 1972).

The error data were averaged across Ss and broken down by display size and type of trial (i.e., positive and negative for marker and no-marker trials). The error rate did not exceed $5 \%$ at any display size for any trial type,
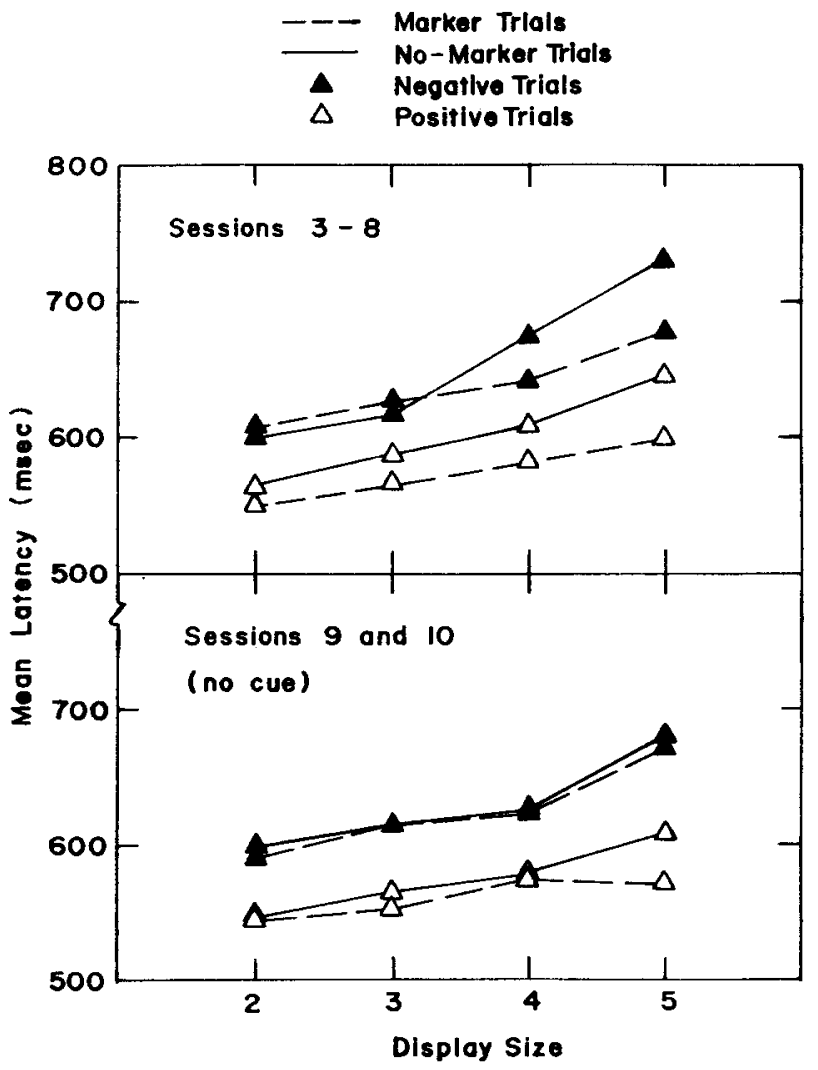

Fig. 1. Mean latencies as a function of display size over Sessions 3-8 and 9-10 (Experiment I).
Table 1

Slopes and Intercepts of Lines Providing Best Fits to Terminal Latency Data Averaged Across Subjects for Experiment I

\begin{tabular}{|c|c|c|c|}
\hline \multicolumn{2}{|c|}{ Trial Type } & Slope & Intercept \\
\hline Marker & $\begin{array}{l}\text { Positive } \\
\text { Megative }\end{array}$ & $\begin{array}{l}16.3 \pm 2.9 \\
22.4 \pm 5.3\end{array}$ & $\begin{array}{l}518.0 \pm 21.5 \\
561.0 \pm 18.4\end{array}$ \\
\hline No Marker & $\begin{array}{l}\text { Positive } \\
\text { Negative }\end{array}$ & $\begin{array}{l}26.4 \pm 5.0 \\
44.9 \pm 4.8\end{array}$ & $\begin{array}{l}509.5 \pm 23.0 \\
497.6 \pm 19.9\end{array}$ \\
\hline
\end{tabular}

Note-Means \pm standard errors in milliseconds.

so it is safe to assume that guessing had no appreciable effect on the observed trends in the latencies. Marker trials showed slightly lower error rates than no-marker trials and no-marker error rates tended to increase with display size, but neither of these effects was statistically significant.

Best-fitting linear functions were found for the curves in Fig. 1. Only the negative no-marker curve showed a significant nonlinear trend. The slopes and intercepts of the best-fitting lines are presented in Table 1, along with their standard errors. 1 Statistical analyses of the obtained slopes and intercepts bear out the above observations concerning Fig. 1. The marker slope for negative trials is significantly less than the negative no-marker slope, $F(1,5)=49.04, p<.01$; the same result holds for the comparison between the positive marker slope and the positive no-marker slope, $F(1,5)=$ $9.98, \mathrm{p}<.05$. For the marker curves, positive and negative slopes are not significantly different, $F(1,5)=$ $1.20, p>.05$. However, the no-marker negative slope is significantly greater than the no-marker positive slope, $\mathrm{F}(1,5)=11.10, \mathrm{p}<.05$.

Turning to intercepts, the difference between marker and no-marker intercepts is significant for negative trials, $\mathrm{F}(1,5)=46.45, \mathrm{p}<.01$, but not for positive trials, $F(1,5)=3.91, p>.05$. Note in Table 1 that the intercepts for marker trials are larger than the no-marker intercepts. The difference in intercepts between positive and negative trials is significant for marker trials, $F(1,5)$ $=6.86, \mathrm{p}<.05$, but not for no-marker trials, $F(1,5)=$ $.36, \mathrm{p}<.05$.

For both marker and no-marker trials, latencies were examined at each display size as a function of the location of the critical letter. While showing no distinct trend, the sets of serial position curves for marker and no-marker trials were quite similar to one another and to those obtained by Atkinson et al (1969). Latencies for negative marker trials were examined as a function of the location of the marker. These serial position curves were quite flat, the points on the curves showing significantly less variability than those for positive marker trials, $F(10,10)=3.77, p<.05$, and positive no-marker trials, $F(10,10)=5.02, p<.05$.

Release data show the same general pattern as terminal data, with the exception that the no-marker negative curve does not deviate significantly from linearity and the slope of the best-fitting line is not 
significantly different from the no-marker positive slope. Also, all release slopes and intercepts are smaller than the corresponding terminal values. One likely reason for the release slopes being smaller is that, on some proportion of the trials at each display size, Ss release the center key before they have completed the search process. Because of the possibility of these permature releases, the release slopes probably underestimate the mean comparison time per item and therefore only the terminal response data are dealt with here.

Not shown in Table 1 are the slopes for the no-cue days. The slopes of the latency-display size functions for the last 2 days of the study are 9.9 and $24.4 \mathrm{msec}$ for positive and negative marker data, respectively, and 19.5 and $25.4 \mathrm{msec}$ for positive and negative no-marker data. Neither the positive nor the negative marker slope is significantly different from the corresponding no-marker slope, $F(1,5)=1.47, F(1,5)=.02$, respectively.

\section{Discussion}

The primary result of this study seems clear. The fact that marker slopes were found to be significantly less than corresponding no-marker slopes suggests that Ss were able to use the marker to, in effect, reduce the number of items processed. Thus, it appears that Ss were able to exercise some control over the search process. However, the fact that the marker slopes were significantly greater than zero shows that the effects of display size were not totally eliminated by the marker.

It is not clear from these data exactly how the marker affected the search processes involved, but the nonzero marker slopes and the fact that the marker intercepts were significantly greater than the no-marker intercepts suggests some possibilities. For example, Ss may have searched the display for the marker, then compared only the marked item with the target item in memory. The slopes of the latency-display size functions for marker trials would then reflect the rate of search for the marker, while the time necessary to compare the marked and target items would result in an increase in the marker intercepts relative to the corresponding no-marker intercepts. Another possibility is that Ss were not able to completely suppress the processing of items in the vicinity of the marker, resulting in an increase in response latency with display size and (as can be shown) an increase in the marker intercepts. Yet another possibility is that Ss were able to restrict processing to the marked item, but simply did not attend to the marker on some proportion of the trials. This also would result in a decrease in slope and an increase in intercept for marker trials relative to no-marker trials. Some of these possibilities will be investigated in Experiment II.

While the significant difference between the two terminal no-marker slopes could be taken as evidence for self-terminating processing, there are a number of reasons for not adopting this interpretation of the no-marker data. First, the difference between the two release no-marker slopes is not significant. The lack of significance could be attributed to a greater number of premature releases on negative than on positive no-marker trials; however, there is no compelling reason why this should occur. Second, in the Atkinson et al study, where the displays were identical to the no-marker displays used here, no such slope difference was found. Third, the distinct nonlinearity of the negative no-marker curve suggests that at the larger display sizes Ss may be "double-checking" before pressing the appropriate key. ${ }^{2}$

If double-checking is the cause of the large slope for the terminal no-marker negative curve, then why is the corresponding marker curve not affected in the same way? Inspection of Fig. 1 shows that there is a slight upturn in the terminal marker negative curve. One obvious explanation for the lack of a larger double-checking effect is that on marker trials Ss simply reprocess the marked item rather than double-checking the whole display.

As noted earlier, on the last 2 days of this study, when Ss were not told which displays contained markers, there were no significant differences between marker and no-marker slopes. Although these results are not based upon enough data to be conclusive, they suggest that $S s$ require prior knowledge that a marker will be in a display in order to use the marker efficiently. In other words, if Ss are not actively searching for the marker, the marker apparently does not "draw attention" to the marked item.

Although Ss were instructed to attempt to use the marker, there was nothing in the design of this study that compelled them to do so. As mentioned above, it is possible that the marker was ignored on some proportion of the marker trials, even when Ss knew which trials were marker triais. In Experiment II, the task used here is modified in order to ensure that Ss use the marker information on every marker trial.

\section{EXPERIMENT II}

In this study, one slight revision is made in the task used for Experiment I. On half of the negative marker trials, the target letter appears among the unmarked items. Thus, on marker trials, the correct response is determined not by the presence or absence of the target letter in the display, but by whether or not the marked item is the target letter.

Besides requiring Ss to attend to the marker, this task also serves to determine whether unmarked items are compared with the letter in memory on marker trials. If the comparison process is carried out on only the marked item, then latencies on negative marker trials should not depend on whether there was a target letter in the display. Differences between latencies on those negative marker trials with a target letter and latencies on those without could be caused only by recognition of the target letter when it appears in the display. 
Fig. 2. Mean latencies as a function of display size over Sessions 3-10 (Experiment II).

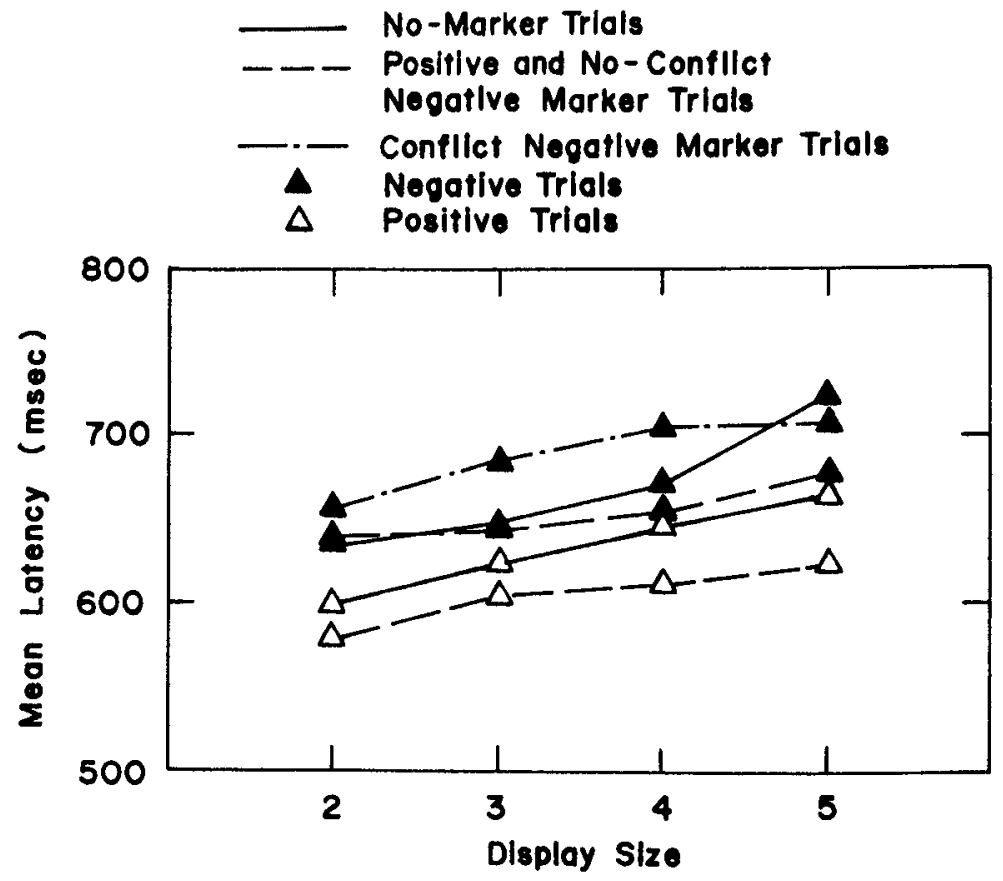

\section{Method}

Subjects. The Ss were six undergraduates obtained through an advertisement in the campus paper. As in the previous study, they all had normal (corrected) vision and were paid $\$ 1.75$ per session.

Procedure. The apparatus, stimuli, and sequence of trial events were identical to those used in Experiment I. The instructions were altered in that Ss were told that on marker trials their task was to determine whether or not the marked consonant was the target letter, while on no-marker trials they were to determine whether or not the target letter appeared anywhere in the display. The selection of the target letter for each trial was changed so that on half of the negative marker trials the target letter would appear in the display but the marker would appear over some other letter. The target letter in this case was chosen randomly from the set of unmarked display items. Ss were informed that each session would contain trials of this type.

As in the previous study, each of the 10 sessions consisted of 8 warm-up trials and 288 data trials, with a short break half-way through the session. However, the light indicating a marker trial was not eliminated during the last two sessions of this study as it had been in the first experiment.

\section{Results}

The data from this study have been divided into five trial types. These are positive and negative no-marker trials, positive marker trials, and two types of negative marker trials, referred to as conflict and no conflict. A conflict negative trial is one in which the target item appears in the display but the marker appears over some other letter. For no-conflict trials, there is a marker in the display but no target letter. Latencies of correct responses for these five trial types are plotted in Fig. 2 as a function of display size. The data are grouped over Ss and Sessions 3-8. A relatively large drop in latencies was observed over the first two sessions, so these sessions were not included in Fig. 2.

As in the previous study, the best-fitting linear function was found for each of the curves in Fig. 2. The slopes and intercepts of these functions are given in Table 2. None of the curves showed significant departure from linearity. The estimated slope of the positive marker curve in Fig. 2 was found to be significantly less than the slope of the corresponding no-marker curve, $F(1,5)=12.60, p<.05$; the same result was obtained when the slope of the no-conflict negative marker curve was compared with the negative no-marker curve, $F(1,5)$ $=11.83, \mathrm{p}<.05$. For both marker and no-marker trials, positive and negative slopes did not differ significantly, $F(1,5)=.08, F(1,5)=3.35$, respectively. Finally, the conflict slopes was not significantly different from the no-conflict slope, $F(1,5)=2.18$.

For intercepts, the following pattern of results was obtained. The difference between the marker and no-marker intercepts was significant for (no-conflict) negative trials, $F(1,5)=9.07, p<.05$, but not for positive trials, $F(1,5)=.01$. For marker trials, the difference between positive and no-conflict negative

Table 2

Slopes and Intercepts of Lines Providing Best Fits to Terminal Latency Data Averaged Across Subjects for Experiment II

\begin{tabular}{|c|c|c|c|}
\hline Trial & Type & Slope & Intercept \\
\hline \multirow{3}{*}{ Marker } & Positive & $14.2 \pm 2.4$ & $555.8 \pm 62.8$ \\
\hline & $\begin{array}{l}\text { Conflict } \\
\text { Negative }\end{array}$ & $17.4 \pm 2.8$ & $627.7 \pm 50.3$ \\
\hline & $\begin{array}{l}\text { No-Conflict } \\
\text { Negative }\end{array}$ & $13.0 \pm 4.0$ & $609.8 \pm 62.3$ \\
\hline No Marker & $\begin{array}{l}\text { Positive } \\
\text { Negative }\end{array}$ & $\begin{array}{l}21.7 \pm 4.0 \\
29.4 \pm 6.0\end{array}$ & $\begin{array}{l}557.0 \pm 74.6 \\
566.1 \pm 61.8\end{array}$ \\
\hline
\end{tabular}

Note-Means \pm standard errors in milliseconds. 
intercepts was significant, $F(1,5)=11.78, p<.05$, while for no-marker trials the positive-negative intercept difference was not significant, $F(1,5)=.41$. The difference between conflict and no-conflict negative marker intercepts was also not significant, $F(1,5)=1.46$.

\section{Discussion}

On the basis of the curves shown in Fig. 2, it appears that compelling Ss to use the marker resulted in no substantial changes in the processing of either marker or no-marker displays. As shown by the tests of significance, the relationships among the slopes found here duplicate those for corresponding curves in the previous experiment, with the exception that the positive and negative no-marker slopes are not significantly different in this study. The absolute magnitudes of the positive slopes in the two studies are also quite similar. In addition, the slopes for no-marker curves found here are very close to those found by Atkinson et al (1969). Their slopes were 23.8 and $26.2 \mathrm{msec}$ for positive and negative trials, respectively, as compared to slopes of 21.4 and $29.4 \mathrm{msec}$ found in this study.

The important new information provided by this study concerns the conflict negative trials. It is evident from the error rates for these trials that Ss were not ignoring the marker. Since each failure to process the marker on a conflict negative trial would lead to an error, the fact that the mean error rate was under $5 \%$ at all display sizes clearly shows that Ss rarely failed to attend to the marker. Turning to response latencies, it is clear from Fig. 2 that while the slopes and intercepts of the conflict negative and no-conflict negative curves do not differ significantly, mean response latencies are slower at every display size for conflict negatives than for no-conflict negatives. Averaging across display size, the mean conflict negative latency is $35 \mathrm{msec}$ greater than the corresponding no-conflict mean, $F(1,5)=$ $31.36, p<.01$. It therefore appears to be the case that on at least some proportion of the marker trials, Ss compare items other than the marked letter with the item in memory; otherwise there would be no difference between conflict and no-conflict latencies. Moreover, an examination of release latencies for negative marker trials shows that the effect of carrying out these comparisons on unmarked items is largely to increase the time between the release and terminal response. For release responses, the mean conflict negative latency (averaged across display size) is only $7 \mathrm{msec}$ greater than the no-conflict mean. This $7-\mathrm{msec}$ difference is significantly less than the $35-\mathrm{msec}$ difference observed for terminal responses, $F(1,5)=15.60, p<.05$.

If the detection of a target letter in the vicinity of the marker results in longer terminal latencies, why are release latencies not similarly affected? The first answer that comes to mind is that $S$ s begin to make a positive response upon detecting both the marker and target letter. Only after releasing the center key do they determine that the target letter is not under the marker. The necessity of ceasing execution of the positive response and making a negative response instead results in a longer terminal latency. However, if this explanation were correct, then the conflict negative release latencies would be close to the release latencies for positive marker trials, the reason being that both types of release would represent the beginning of a positive response. But conflict negative release latencies are closer to the no-conflict negative than to the positive release latencies. This finding suggests that the conflict negative release represents the beginning of a negative, not a positive response. If this is the case, then the target letter in the display must not be compared with the target item in memory until after the marked letter has been compared, with execution of the negative response being slowed by detection of the target letter.

\section{SUMMARY}

The data in Experiments I and II are most easily explained by assuming that on marker trials Ss first locate the marker (perhaps by means of a search process having a duration that increases with display size). Upon locating the marker, they compare the marked letter with the target in memory; depending on the outcome of this comparison, they then initiate either a positive or a negative response. However, the comparison process is continued with other letters in the vicinity of the marker. If a negative response has been initiated and the target letter is detected in one of these subsequent comparisons, the negative response is delayed, perhaps until a portion of the display has been reprocessed. This explanation is similar in some respects to the above-mentioned interpretation given by Eriksen and Hoffman (1972a, b, 1973) of their data. Recall that they concluded that everything within $1 / 2$ deg on either side of the marked item was processed in detail. As mentioned above, in the present study, conflict negative latencies were much slower when the target letter was adjacent to the marked letter than when in any other nonadjacent position. Since only the letters adjacent to the marked item were within $1 / 2 \mathrm{deg}$ of the marker, this finding is consistent with the conclusion of Eriksen and Hoffman that detailed processing occurs in an area of about that size.

\section{REFERENCES}

Atkinson, R. C., Holmgren, J. E., \& Juola, J. F. Processing time as influenced by the number of elementsin a visual display. Perception \& Psychophysics, 1969, 6, 321-326.

Averbach, E., \& Coriell, A. S. Short-term memory in vision. Bell System Technical Journal, 1961, 40, 309-328.

Eriksen, C. W., \& Hoffman. J. E. Some characteristics of selective attention in visual penception determined by vocal reaction time. Perception \& Psychophysics, 1972a, 11, 169-171.

Eriksen, C. W. \& Hoffman, J. E. Temporal and spatial characteristics of selective encoding from visual displays. Perception \& Psychophysics, 1972b, 12, 201-204. 
Eriksen, C. W., \& Hoffman, J. E. The extent of processing of noise elements during selective encoding from visual displays. Perception \& Psychophysics, 1973, 14, 155-160.

Nickerson, R. S. Binary-classification reaction time: A review of some studies of human information-processing capabilities. Psychonomic Monograph Supplements, 1972, 4(17, Whole No. 65), 275-318

Rumelhart, D. E. A multicomponent theory of the perception of briefly exposed visual displays. Journal of Mathematical Psychology, 1970, 7, 191-218.

Sperling, $G$. The information available in brief visual displays. Psychological Monographs, 1960, 74(11, Whole No. 498).

\section{NOTES}

1. The slopes and intercepts of best-fitting lines were found for each $S$; the standard errors given in Table 2 are the standard errors of the means of these values across individuals.

2. It could be argued that the observed nonlinearity is due to eye movements at the larger display sizes. The exposure time of $400 \mathrm{msec}$ is sufficiently long for an eye movement to take place before offset of the display. However, previous work with this apparatus (Atkinson et al, 1969) has shown that results obtained with this exposure time are essentially the same as those obtained with an exposure time of $150 \mathrm{msec}$.

(Received for publication July 2,1973 ; revision accepted January 28,1974 .) 